\title{
A CONSTANT REGRESSION CHARACTERIZATION OF THE GAMMA LAW
}

\author{
JACEK WESOŁOWSKI, ${ }^{*}$ Politechnika Warszawska
}

\begin{abstract}
A constant regression of the quotient on the sum of two i.i.d. nondegenerate positive random variables is a characteristic property of the gamma distribution.
\end{abstract}

In Lukacs (1955) the following nice characterization of the gamma law has been obtained: if random variables $X, Y$ are independent non-degenerate and positive then the random variables $X / Y$ and $X+Y$ are independent iff $X$ and $Y$ have gamma distributions. This result has been the starting point of numerous investigations. Many of them are listed in Wang (1981) and in Shanbhag's review of this paper in Mathematical Reviews. Some more recent contributions are by Khatri (1984), Dilip (1984), (1985), Sathe and Dixit (1985), Letac (1985), Sim (1986) and Wesołowski (1989).

Among regressional versions of Lukacs' theorem, considered by many authors, the most important seems to be the characterization using conditions

$$
E(X \mid X+Y)=a(X+Y), \quad E\left(X^{2} \mid X+Y\right)=b(X+Y)^{2},
$$

where $a, b$ are real numbers and $X, Y$ are independent non-degenerate positive random variables.

In this note we replace Lukacs' independence condition by other regressional assumptions.

Theorem 1. Let $X$ and $Y$ be independent non-degenerate positive random variables such that $E X<\infty$ and $E X^{-1}<\infty$. If the conditions

$$
\begin{gathered}
E(X \mid X+Y)=a(X+Y), \\
E\left(X^{-1} \mid X+Y\right)=b(X+Y)^{-1}
\end{gathered}
$$

hold for some real $a, b$, then $0<b^{-1}<a<1$ and $X, Y$ have gamma distributions with the same scale parameter.

Proof. From the assumptions we get

$$
\begin{gathered}
(1-a) E X=a E Y, \quad E X^{-1}=b E(X+Y)^{-1}, \\
E Y E X^{-1}=b-1 .
\end{gathered}
$$

Consequently $0<a<1, b>1$. The non-degeneracy of $X$ implies $E X E X^{-1}>1$ and $a b>1$.

For $t<0$, introduce $h(t)=E X^{-1} \exp (t X)$ and $f(t)=E \exp (t Y) ; h, f$ and all their derivatives are strictly positive on $(-\infty, 0)$. Denoting $k=E X^{-1}$, the limits of $h, h^{\prime}$ and $f$ are $k, 1$ and 1 when $t \uparrow 0$.

Received 8 December 1989; revision received 6 February 1990.

* Postal address: Instytut Matematyki, Politechnika Warszawska, Plac Jedności Robotniczej 1, 00-661 Warszawa, Poland. 
From (1) and (2) we have, respectively,

Hence

$$
\begin{gathered}
(1-a) h^{\prime \prime}(t) f(t)=a h^{\prime}(t) f^{\prime}(t), \\
h(t) f^{\prime}(t)=(b-1) h^{\prime}(t) f(t) .
\end{gathered}
$$

$$
\frac{f^{\prime}}{f}=(b-1) \frac{h^{\prime}}{h}=\frac{(1-a) h^{\prime \prime}}{a h^{\prime}} .
$$

Denote $c=a(b-1) /(1-a)$. Observe that $c-1=(a b-1) /(1-a)>0$. From (3)

$$
h^{\prime}(t)=\left(k^{-1} h(t)\right)^{c},
$$

which implies

and consequently

$$
(h(t))^{1-c}=k^{-c}(t(1-c)+k)
$$

$$
E \exp (t X)=h^{\prime}(t)=\left(1-\frac{t(c-1)}{k}\right)^{-c /(c-1)}
$$

Now from the first equation in (3) we get

$$
E \exp (t Y)=\left(1-\frac{t(c-1)}{k}\right)^{-c(1-a) / a(c-1)} .
$$

Hence $X$ and $Y$ have gamma distributions with the same scale parameter $k /(c-1)$ and shape parameters

respectively.

$$
\frac{a(b-1)}{a b-1} \text { and } \frac{(b-1)(1-a)}{a b-1}
$$

As a simple corollary from Theorem 1 we have the following characterization for identically distributed random variables.

Theorem 2. Let $X$ and $Y$ be i.i.d. non-degenerate positive random variables such that $E X<\infty$ and $E X^{-1}<\infty$. If

$$
E(Y / X \mid X+Y)=c
$$

for some real number $c$ then $c>1$ and the random variables $X, Y$ have a gamma distribution.

Proof. The assumption of identical distributions yields (1) with $a=1 / 2$. On the other hand (4) implies (2) with $b=c+1$. Consequently $1>a b=(c+1) / 2$ and thus the result follows from Theorem 1.

\section{Acknowledgement}

Application of Laplace transforms, suggested by the referee, instead of characteristic functions as used in the original version, eliminated various unnecessary subtleties.

\section{References}

Dilip, R. (1984) A characterization of the generalized gamma distribution. Calcutta Statist. Assoc. Bull. 33, 137-141.

Dilip, R. (1985) Addendum to 'A characterization. . '. Calcutta Statist. Assoc. Bull. 34, 125.

KHATRI, C. G. (1984) Characterization of gamma and $G$-binomial distributions based on regressions. J. Indian Statist. Assoc. 22, 115-128.

Letac, G. (1985) A characterization of the gamma distribution. Adv. Appl. Prob. 17, 911-912.

LuKACs, E. (1955) A characterization of the gamma distribution. Ann. Math. Statist. 26, 319-324. 
SATHE, Y. S. AND DiXrt, U. J. (1985) A comment on generalized gamma distribution. Calcutta Statist. Assoc. Bull. 34, 123-124.

SiM, C. H. (1986) Characterization of the gamma distribution. Bull. Malaysian Math. Soc. 9, 81-84.

WANG, Y. H. (1981) Extensions of Lukacs' characterization of the gamma distribution. In Analytical Methods in Probability Theory (Proceedings, Oberwolfach, Germany, 1980), Lecture Notes in Mathematics 861, Springer, New York, 166-177.

WESOŁOWSKI, J. (1989) A characterization of the gamma process by conditional moments. Metrika 36, 299-309. 